\title{
Le rapport État-religions au Japon et ses portées juridiques
}

\section{Moe Wada}

\section{(2) OpenEdition}

\section{Journals}

Édition électronique

URL : https://journals.openedition.org/rdr/1458

DOI : $10.4000 /$ rdr. 1458

ISSN : 2534-7462

Éditeur

Presses universitaires de Strasbourg

\section{Édition imprimée}

Date de publication : 6 mai 2021

Pagination : 155-170

ISBN : 979-10-344-0089-8

ISSN : 2493-8637

Référence électronique

Moe Wada, «Le rapport État-religions au Japon et ses portées juridiques », Revue du droit des religions [En ligne], 11 | 2021, mis en ligne le 06 mai 2021, consulté le 02 mai 2022. URL : http:// journals.openedition.org/rdr/1458; DOI : https://doi.org/10.4000/rdr.1458

\section{(c) $(1)(9$}

La revue du droit des religions est mise à disposition selon les termes de la Creative Commons Attribution - Pas d'Utilisation Commerciale 4.0 International - CC BY-NC 4.0. 


\section{Le rapport État-religions au Japon et ses portées juridiques}

\section{Moe WADA}

Doctorante en sciences humaines et de l'environnement, Université de Kyoto

\section{Résumé}

Cet article vise à analyser le principe constitutionnel de séparation entre l'État et les religions au Japon ainsi que sa portée juridique à travers une étude empirique. Il s'agit à la fois d'une introduction au contexte historique et culturel japonais en matière religieuse et d'une étude de cas récents. En particulier, l'accent est mis sur le modèle japonais de «laïcité» et sur la façon dont le principe de séparation est appliqué dans le but de satisfaire aux demandes religieuses minoritaires qui seront potentiellement sources de ressources économiques, notamment grâce au tourisme, pour la société japonaise.

\section{Abstract}

This article aims to analyse the constitutional principle of the separation between state and religions in Japan as well as its legal scope through an empirical study. It is an introduction to the Japanese historical and cultural context of religious issues as well as a recent case study on this matter. In particular, we will focus on the Japanese model of «laicité » and how the principle of the separation applies in order to meet minority religious demands that will potentially be sources of economic resources for Japanese society, notably through tourism. 


\section{INTRODUCTION}

Le Japon compte entre 100000 et 200000 habitants de religion musulmane seulement pour environ 126 millions de Japonais, même en prenant en compte les résidents étrangers ${ }^{1}$. Cependant, dans un contexte d'accroissement du nombre de visiteurs venant d'Asie du Sud-Est, la société japonaise se trouve confrontée à la question des mesures et dispositions susceptibles de répondre à d'éventuelles demandes religieuses. Selon les statistiques réalisées par l'Office national du tourisme japonais (JNTO), au cours de ces dernières années, le nombre de touristes venant de pays membres de l'Association des nations de l'Asie du Sud-Est (ANASE) augmente, tout comme de ceux venant d'Indonésie, de Malaisie et de Singapour, des pays à forte population musulmane ${ }^{2}$. Selon le rapport rédigé par le Bureau de planification stratégique du tourisme au sein de l'Agence du tourisme japonais (kankô-chô), un des motifs principaux de ces voyages est le désir de découvrir la cuisine japonaise. Les touristes musulmans rencontrent toutefois souvent des difficultés liées à leurs pratiques religieuses: il reste compliqué de trouver des produits halal ainsi que des lieux de prière. Cela a conduit des touristes musulmans et des agences de voyages à demander des aménagements permettant de voyager en pratiquant sa religion ${ }^{3}$. Depuis 2015, le gouvernement japonais a décidé d'engager des actions dans le but de surmonter ces difficultés, avec comme objectif de développer le marché touristique musulman.

1. Aucun chiffre officiel n'existe mais des chercheurs ont proposé des estimations. V. H. TANADA, «Sekai to Nihon no Musulimu Jinkou 2018 nen» [La population musulmane dans le monde et au Japon, 2018], NingenKagaku Kenkyu, vol. 32 (2), 2019, p. 253-262. Selon Tanada, le nombre de musulmans étrangers ayant le droit de résidence est d'environ 157000 , celui des musulmans japonais d'environ 43000 au Japon.

2. En 2008, le nombre de passagers venant d'Indonésie était environ de 66000, 105000 de la Malaisie et 167000 de Singapour. Dix ans après, en 2018, les chiffres passent à 396000 pour l'Indonésie, 468000 pour la Malaisie et 437000 pour Singapour (JAPAN NATIONAL TOURISM ORGANISATION, «Trends in annual Visitor Arrivals to Japan by Country/Area » : https://statistics.jnto.go.jp/en/graph/\#graph--trends--by--country [consulté le 7 déc. 2020]). Selon une autre statistique, la population musulmane, en 2010 , représente $88,1 \%$ de la population en Indonésie, 61,4 \% en Malaisie et 14,9\% à Singapour (Pew Research Center, "The Future of the Global Muslim Population», janv. 2011: www.pewforum.org/wp-content/ uploads/sites/7/2011/01/FutureGlobalMuslimPopulation-WebPDF-Feb10.pdf [consulté le 7 déc. 2020]).

3. Force opérationnelle pour la promotion de stratégie touristique, «Plan d'action pour des mesures en faveur des touristes musulmans", mai 2018, disponible en japonais: www.kantei.go.jp/jp/singi/kanko_vision/pdf/h300522actionplan_honbun.pdf [consulté le 7 déc. 2020]. 
Cet article ${ }^{4}$ se propose d'examiner la conformité de ces mesures prises au bénéfice des touristes musulmans avec le principe constitutionnel de séparation entre l'État et les religions au Japon. Comme nous le verrons, le principe de séparation au Japon a un lien historique fort avec le shintoïsme, une des religions traditionnelles du Japon. Par conséquent, bien que la littérature et les jurisprudences sur la constitutionnalité des rapports entre l'État et le shintoïsme soient assez abondantes, on ne peut pas en dire autant pour les religions des nouveaux arrivants. Il y a nécessité à mettre en valeur la portée du principe de séparation entre l'État et les religions au Japon, en particulier à l'égard des touristes ayant des demandes religieuses. L'islam, que nous examinerons ici, reste minoritaire au Japon, mais est considéré comme un élément important dans le domaine du tourisme. Cet article propose donc une analyse de la forme japonaise des rapports État-religions, à travers quelques exemples, et tente d'esquisser la possibilité d'un modèle japonais de «laïcité». Notre but n'est pas de présenter une comparaison entre le modèle français de laïcité et le modèle japonais, mais de montrer que la question de la laïcité est aussi importante au Japon - un pays dont la diversité s'accentue - qu'en France.

Dans une première partie, nous présenterons la «laïcité» dans le système japonais à partir de l'histoire de la mise en ouvre du principe de séparation entre l'État et les religions au Japon, en particulier le rapport de l'État avec le shintoïsme d'État (kokka shintô). La nouvelle Constitution introduite par les États-Unis a ouvert une voie à la séparation stricte de l'État et des religions. Nous verrons ensuite les jurisprudences de la Cour suprême relatives au principe de séparation.

Dans une seconde partie, nous présenterons plusieurs mesures prises en faveur des touristes, pour lesquelles un investissement public pour satisfaire les demandes religieuses a été effectué. Nous éclairerons dans quelle mesure la légitimité et la constitutionnalité de ces mesures ont été déduites par certaines collectivités locales et l'Office national du tourisme japonais, en se référant à des cas empiriques.

4. Cet article a été rédigé avec le soutien de la Société japonaise pour la promotion de la science (JSPS) n JP19J23056. Je remercie également Simon Serverin (Université Sophia, Tokyo) pour sa relecture. 


\section{LE RAPPORT ÉTAT-RELIGIONS AU JAPON}

\subsection{LE MODÈLE JAPONAIS DE "LAÏCITÉ »}

Lorsque nous décrivons le système des rapports État-religions au Japon, est-il pertinent d'adopter le mot «laïcité»? La laïcité est un terme français dont le sens a connu de nombreuses variations. Le concept a évolué au sein de la société française et a été l'objet de débats passionnés. En France, avec la croissance de la visibilité religieuse dans l'espace public, cette notion ne peut plus être réduite au simple principe de séparation entre les Églises et l'État, établi par la loi de 1905. Par exemple, le principe de laïcité a été invoqué lors de l'adoption de la loi du 15 mars 2004 encadrant, en application du principe de laicité, le port de signes ou de tenues manifestant une appartenance religieuse dans les écoles, collèges et lycées publics. Le débat politique sur le principe de laïcité s'est aussi enflammé lorsque s'est posée la question de la liberté d'expression religieuse des salariés des employeurs de

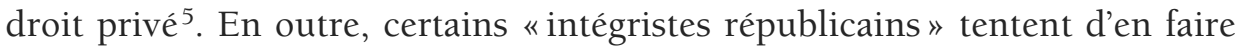
le fer de lance des valeurs nationales républicaines, avec l'islam en ligne de mire $^{6}$. La notion de laïcité française a donné lieu à de vives controverses.

Sur le plan juridique français, les références légales de la laïcité se sont déplacées du principe de séparation établi par la loi de 1905 au principe de neutralité consacré par la Constitution. Selon David Koussens, face à la nécessité de réguler la diversité religieuse, «le principe de neutralité se trouve

5. En 2011, le Haut Conseil à l'intégration a proposé dans un avis l'extension du principe de laïcité aux structures privées chargées d'une mission de service public ou d'intérêt général: HCI, Expression religieuse et laïcité dans l'entreprise, ${ }^{\text {er }}$ sept. 2011 : http://archives.hci.gouv. fr/IMG/pdf/HCI-Avis-laicite-entreprise-pdf-2.pdf [consulté le 7 déc. 2020]. Ce débat est lié à l'affaire Baby-Loup, dans laquelle le licenciement d'une employée portant le voile islamique est mis en cause. La chambre sociale de la Cour de cassation précise que «le principe de laïcité instauré par l'article $1^{\text {er }}$ de la Constitution n'est pas applicable aux salariés des employeurs de droit privé qui ne gèrent pas un service public»: Cass. soc., 19 mars 2013, $\mathrm{n}^{\circ}$ 11-28.845. L'Assemblée plénière de la Cour de cassation réaffirme cette doctrine: Cass. ass. plén., 25 juin 2014, $\mathrm{n}^{\circ}$ 13-28.369. Dans le cadre législatif, la loi $\mathrm{n}^{\circ}$ 2016-1088 du 8 août 2016 a inséré dans le Code du travail l'article L. 1321-2-1 autorisant des clauses du règlement intérieur qui inscrivent le principe de neutralité et restreignent la manifestation des convictions des salariées sous certaines conditions. L'Observatoire de la laïcité et la CNCDH estiment que cet article s'oppose au principe de laïcité et ouvre la voie à d'éventuelles discriminations et au développement d'entreprises communautaires: www. cncdh.fr/sites/default/files/160719_cp_art_1_pjl_travail_avec_observatoire_laicite_vdef.pdf [consulté le 7 déc. 2020].

6. J. BAubÉROt, L’intégrisme républicain contre la laïcité, La Tour d'Aigues, Éditions de l'Aube, 2006, p. 67-70. 
aux fondements de la laïcité ${ }^{7} »$. Jean-Marie Woehrling souligne également que «le principe de séparation est apparu moins significatif que le principe de neutralité ${ }^{8} »$ depuis les années 2000. Dans sa décision du 19 novembre 2004 relative au Traité constitutionnel de l'Europe, le Conseil constitutionnel se réfère au principe de laïcité sans invoquer le principe de séparation ${ }^{9}$. Par ailleurs, le Conseil d'État définit dans son rapport en 2004 que la laïcité française doit se décliner en trois principes: ceux de neutralité de l'État, de liberté religieuse et de respect du pluralisme ${ }^{10}$.

Alors que la notion juridique de laïcité se réfère tantôt au principe de séparation tantôt au principe de neutralité, D. Koussens affirme que le discours sur la laïcité en France ne correspond pas toujours à la réalité juridique de la laïcité ${ }^{11}$. L'idée sociologique contribue donc à éclairer les interprétations diverses du discours sur la laïcité. Jean Baubérot et Micheline Milot proposent ainsi des idéaux types de la laïcité pertinents comme outils d'analyse, permettant d'appréhender les phénomènes que nous observons dans la réalité historique et sociale, à partir des caractéristiques théoriquement élaborées ${ }^{12}$.

Comme l'a souligné Susumu Shimazono, la laïcité est le fruit du modèle chrétien occidental et il ne serait pas rationnel d'appliquer cette notion à d'autres contextes nationaux où le politique et le religieux ne sont pas forcément nettement dissociés ${ }^{13}$. Pour autant, cela ne nous empêche pas d'analyser le modèle japonais des rapports entre l'État et les religions à partir des idéaux types de laïcité et de ses éléments constitutifs. C'est ce que Kiyonobu Daté a exactement entrepris d'étudier, afin d'élaborer le concept de laïcité dans un dialogue entre civilisations ${ }^{14}$. Il relève que la Constitution du Japon de 1946 prône une laïcité séparatiste très radicale, que nous examinerons ci-dessous. Depuis

7. D. Koussens, L'épreuve de la neutralité. La laïcité française entre droits et discours, Bruxelles, Bruylant, 2015, p. 8.

8. J. M. Woenrling, «Le principe de neutralité confessionnelle de l'État», Société, droit et religion, $\mathrm{n}^{\circ} 1,2011$, p. 63-85.

9. Décision no 2004-505 DC du 19 nov. 2004 relative au Traité établissant une Constitution pour l'Europe.

10. Conseil D'État, Rapport public. Un siècle de laïcité, Paris, La Documentation française, 2004, p. 272.

11. D. Koussens, op. cit., p. 9.

12. J. Baubérot, M. Milot, Laïcités sans frontières, Paris, Seuil, 2011.

13. S. Shimazono, «Nihon no Sezokuka to Syukyo Gainen» [La laïcisation et la notion de religion au Japon], in M. HANEDA et al. (dir.), Sécularisations et laïcités, Tokyo, University of Tokyo, 2009, p. 83-89, disponible en français, p. 71-78.

14. K. DAtÉ, «De la laïcité de séparation à la laïcité de reconnaissance au Japon ? », in J. BAUBÉROT et al. (dir.), Laïcité, laïcités: Reconfigurations et nouveaux défis, Paris, Éditions de la Maison des sciences de l'homme, 2015, p. 169-188. 
la mise en œuvre de la Constitution de 1946 (entrée en vigueur en 1947), l'État s'interdit d'exercer toute activité religieuse et d'octroyer des privilèges à une religion particulière. Bien que le mot «laïcité» ne soit pas explicitement présent dans les textes juridiques et constitutionnels, le Japon pourrait être considéré comme un État laïque tant que la garantie de la liberté religieuse et la neutralité de l'État en matière religieuse seront assurées par la Constitution.

\subsection{HISTOIRE DE LA SÉPARATION DE L'ÉTAT ET DES RELIGIONS AU JAPON}

Lorsque la séparation de l'État et des religions prête à discussion au Japon, il s'agit essentiellement de problèmes avec le shintoïsme. Une étude historique révèle que le shintoïsme a joué un rôle important dans les affaires politiques du Japon. Après la Restauration de Meiji au XIX $x^{e}$ siècle, les dirigeants firent du shintoïsme la religion officielle, établissant le shintoïsme d'État, et les temples shinto ont bénéficié d'un statut privilégié par rapport aux autres religions. Le but était de donner une légitimité politique à l'Empereur, car la famille impériale était considérée comme descendante d'Amaterasu, déesse du soleil dans le shintoïsme. Sous le régime du shintoïsme d'État, le gouvernement a renforcé le patriotisme et la loyauté du peuple japonais envers l'Empereur, en l'obligeant à pratiquer le shintoïsme d'État. Pendant la seconde guerre mondiale, le shintoïsme d'État exhortait non seulement les soldats, mais toute la population japonaise à lutter pour l'Empereur ${ }^{15}$. Cependant, la liberté religieuse était aussi inscrite dans la Constitution de l'Empire du Japon de 1889, dont l'article 28 énonce que « tout sujet japonais jouira de la liberté de religion, dans les limites compatibles tant avec ses devoirs de sujet qu'avec l'ordre et la paix publique». Le Gouvernement avait dû répondre à la résistance des bouddhistes et aux demandes des pays occidentaux qui exigeaient l'autorisation des missions chrétiennes et l'institutionnalisation de la liberté religieuse ${ }^{16}$.

Après la seconde guerre mondiale, le plan américain a visé à la démilitarisation complète du Japon pendant la première période de l'occupation et à la mise en place d'une démocratie modernisée ${ }^{17}$. La séparation entre l'État et le shintoïsme était indispensable tant pour l'élimination de l'ultranationalisme et du militarisme japonais que pour la garantie de la liberté

15. J. M. Kitagawa, Religions in Japanese History (American Lectures on the History of Religions), New York, Columbia University Press, 1990, p. 269.

16. S. Shimazono, art. cit., p. 83-84, en français p. 72.

17. J. M. Kitagawa, op. cit., p. 262 
religieuse. Au cours de l'occupation du Japon, le Commandement suprême des forces alliées (SCAP) a rédigé la «directive shinto», visant à supprimer le shintoïsme d'État ${ }^{18}$. Il a ensuite démantelé le régime du shintoïsme d'État par la promulgation de la nouvelle Constitution de 1946, qui établissait juridiquement la liberté de religion. La nécessité d'éliminer le shintoïsme d'État afin de prévenir un retour de l'ultranationalisme a conduit l'armée américaine à rédiger la nouvelle Constitution qui prévoyait la séparation complète de l'État et des religions. L'État se voit ainsi imposer la neutralité en matière religieuse par la Constitution de 1946, dont les articles 20 et 89 assurent la liberté de conscience et de religion et interdisent le financement des religions ${ }^{19}$.

En effet, la Constitution de 1946 avait pour but d'éliminer l'influence du shintoïsme de la sphère publique et d'affranchir l'État d'une confession dominante. En ce sens, le système des rapports État-religions au Japon peut être analysé comme une «laïcité séparatiste», radicalisant la rupture entre la sphère publique et la sphère privée. Selon J. Baubérot et M. Milot, la séparation est un moyen pour garantir la liberté de conscience et l'égalité entre les cultes, mais lorsque la séparation apparaît comme une finalité en soi, il s'agit d'un type de laïcité séparatiste. Dans un type séparatiste de laïcité, la séparation devient une norme «surdéterminante» dans l'interprétation conférée aux trois autres principes (liberté de conscience, égalité et neutralité) ${ }^{20}$. Dans la réalité juridique, cependant, nous constatons que l'interprétation et l'application de ce principe de séparation par la Cour suprême ne sont pas aussi strictes. Dans les décisions relatives au principe de séparation, qui seront examinées ci-dessous, la Cour s'est toujours montrée assez tolérante à l'égard de l'État: a contrario de la séparation complète de l'État et des religions prévue par la Constitution, la Cour suprême japonaise porte un regard bienveillant lorsque le gouvernement s'investit dans les affaires du

18. Supreme Commander for Allied Powers, SCAPIN-448, Abolition of Govermental Sponsorship, Support, Perpetuation, Control and Dissemination of State Shinto (Kokka Shinto, Jinja Shinto), 15 Dec. 1945: https://dl.ndl.go.jp/info:ndljp/pid/9885515 [consulté le 7 déc. 2020].

19. «La liberté de croyance religieuse est garantie à toute personne. Aucun groupement religieux ne peut être privilégié par l'État pas plus qu'il n'exerce le pouvoir politique. Nul n'est contraint de participer aux actes, aux cérémonies, aux cultes ou aux manifestations d'ordre religieux. L'État et ses organes n'ont pas la permission de donner un enseignement religieux ni d'exercer d'autres activités religieuses. » [Art. 20] ; «Le fonds public et les autres domaines publics ne peuvent être affectés ou employés en vue de faciliter l'emploi, le bénéfice ou le maintien des institutions ou associations religieuses ni au profit des entreprises de charité, d'éducation ou de fraternité qui ne se trouvent pas sous le contrôle public.» [Art. 89].

20. J. Baubérot, M. Milot, Laïcités sans frontières, op. cit., p. 90. 
shintoïsme ${ }^{21}$. L'interprétation que propose Yoichi Koizumi de ce paradoxe est que si la Constitution pose une définition stricte de la séparation, elle ne montre pas d'hostilité à l'égard des religions: elle a pour but de démocratiser la politique japonaise, de protéger son pacifisme et d'assurer pleinement la liberté religieuse ${ }^{22}$.

\subsection{LA JURISPRUDENCE RELATIVE À LA SÉPARATION DES RELIGIONS DE L'ÉTAT}

Les premières jurisprudences éclairent l'application du principe de séparation au Japon. Le premier litige concernait l'utilisation de fonds publics par une municipalité pour une cérémonie shintoïste, le jichinsai (Kakunaga $v$. Sekiguchi en 1977) ${ }^{23}$. Le jichinsai est une cérémonie religieuse préalable à la construction des bâtiments, qui a pour objectif d'assurer la sécurité des travaux par une prière selon des rites shintoïstes. Une municipalité avait présidé une telle cérémonie organisée pour la construction d'un gymnase public et la constitutionnalité de cette cérémonie a été questionnée. Le tribunal de première instance a déclaré que l'acte de la municipalité était constitutionnel, en considérant ce rituel comme une coutume sans caractère religieux. La cour d'appel l'a en revanche jugé inconstitutionnel, en insistant sur sa signification religieuse. Enfin, la Cour suprême a jugé qu'une séparation totale entre l'État et la religion était en pratique impossible et que les activités religieuses interdites par l'article 20 alinéa 3 de la Constitution désignaient seulement des actes qui dépassaient la limite raisonnable. Cette limite a été clarifiée selon le «critère de l'objectif et de l'effet», une référence directe à la jurisprudence américaine du Lemon test que la Cour suprême des États-Unis a introduit dans l'arrêt Lemon v. Kurtzman en 1971. Sur cette base théorique, la Cour suprême du Japon a expliqué que le principe constitutionnel de séparation n'interdisait pas tout contact étatique avec la religion, malgré l'obligation de neutralité de l'État en matière religieuse. L'article 20 alinéa 3 interdit les actes de l'État dont le but est religieux et qui apportent, par leurs effets, assistance ou aide à des religions, les promeuvent ou exercent des pressions et interventions vis-à-vis d'elles. La Cour

21. S. Matsui, «Japan: The Supreme Court and the separation of church and state», International Journal of Constitutional Law, vol. 2(3), 2004, p. 534-545.

22. Y. Koizumi, «Laïcité et liberté religieuse au Japon», Konan Journal of social sciences, vol. 7, 2000, p. 18.

23. Saikô Saibansho [Cour suprême], 13 juill. 1977: Recueil civil, vol. 31, no 4, p. 533, disponible en anglais: https://www.courts.go.jp/app/hanrei_en/detail?id=51 [consulté le 7 déc. 2020]. V. aussi, Y. Koizumi, «Les rapports État-religions au Japon et la laïcité», RDP 2011, p. 1641-1657, spéc. p. 1653. 
ne nie pas que la cérémonie présidée par la municipalité a des liens avec une religion, mais elle considère que cette cérémonie est un simple rituel social séculier ayant pour but de souhaiter la sécurité des ouvriers lors des travaux de construction. En conséquence, la Cour a conclu que l'activité de la municipalité n'était pas une activité religieuse interdite par l'article 20 et qu'elle était conforme à la Constitution.

Le deuxième litige à mentionner concerne la présence du maire de Hakusan, une municipalité d'Ishikawa, à une cérémonie de soutien pour une fête d'anniversaire de la consécration d'un temple shintoïste (Shirayama Hime Jinja). La cérémonie présidée par une association ayant pour but d'organiser la fête a été vue comme relevant d'une activité religieuse et a suscité, par conséquent, un débat sur la constitutionnalité de l'initiative du maire. La cour d'appel a jugé inconstitutionnelle la participation du maire à la fêteanniversaire de la consécration du temple, car l'objectif de celle-ci avait une signification religieuse et apportait, par son effet, assistance et aide à une religion. De même que dans l'affaire Kakunaga v. Sekiguchi, le jugement de la cour d'appel se réfère explicitement au critère de l'objectif et de l'effet. À la suite du pourvoi, la Cour suprême a pu se prononcer sur la constitutionnalité de l'initiative du maire. Considérant globalement les circonstances et faisant référence aux conditions sociales et culturelles du Japon, la Cour n'a pas jugé que le rapport de l'acte mentionné avec la religion dépassait la limite raisonnable ${ }^{24}$. Soulignant l'importance touristique de la fête du temple, la Cour a confirmé la constitutionnalité de la présence du maire - dont le rôle était de prendre part au développement du tourisme dans cette région - et de l'utilisation de fonds publics.

Si nous ne pouvons examiner tous les litiges relatifs au principe de séparation, ces exemples permettent de relever deux points du système des rapports entre l'État et les religions. Premièrement, le principe constitutionnel de séparation désigne par nature une séparation stricte entre l'État et les religions. De ce point de vue, le modèle japonais de «laïcité» appartient à une laïcité séparatiste, insistant sur la séparation complète. Cependant, les décisions judiciaires sur la constitutionnalité des actes de l'État et des collectivités locales adoptent une interprétation moins stricte de ce principe. En se référant au critère de l'objectif et de l'effet, selon lequel les actes ayant des liens avec une religion ne sont pas en eux-mêmes considérés comme

24. Saikô Saibansho [Cour suprême], 22 juill. 2010 : Recueil civil, $\mathrm{n}^{\circ}$ 234, p. 337, disponible en japonais: http://www.courts.go.jp/app/files/hanrei_jp/471/080471_hanrei.pdf [consulté le 7 déc. 2020]. 
inconstitutionnels, les décisions prennent en compte leur signification, leur objectif et leur effet.

Deuxièmement, si le principe constitutionnel de séparation est appliqué d'une manière plus souple que le prévoient les dispositions de la Constitution, le modèle japonais de "laicité» pourra être plus ouvert qu'une laïcité séparatiste. Cela permet à l'État de prendre des mesures pragmatiques concernant les actes qui ont des liens avec des religions. Bien qu'historiquement la séparation stricte ait été conçue comme une finalité indispensable, cela ne signifie pas que le modèle japonais de "laïcité» soit synonyme d'hostilité à l'égard de toute activité étatique ayant des liens avec une religion. Dans ce modèle, il s'agit de la neutralité de l'État en matière religieuse. En ce sens, le modèle de "laïcité» au Japon s'approche de celui de la laïcité française, qui met l'accent sur le principe de neutralité plus que sur le principe de séparation.

\section{LA PORTÉE DU PRINCIPE DE SÉPARATION FACE AUX MINORITÉS RELIGIEUSES}

\subsection{LA NÉCESSITÉ DE LA MISE EN PLACE DE DISPOSITIFS EN FAVEUR DES MUSULMANS}

Nous avons présenté le modèle japonais de «laïcité» et sa portée juridique, assouplie par les interprétations de la Constitution par la justice japonaise. La question se pose, dans un second temps, de sa portée pour la minorité musulmane. Au Japon, les mesures répondant efficacement aux demandes religieuses telles que la construction de salles de prière et l'accès aux produits halal restaient limitées jusqu'aux années 2010. Comme nous l'avons évoqué plus haut, de nombreux touristes musulmans visitent le Japon en provenance d'Indonésie, de Malaisie et de Singapour. Cela pose des problèmes concernant leur pratique religieuse. Depuis les années 2010, certaines collectivités locales et entreprises privées ont commencé à organiser des séminaires pour que les participants puissent approfondir leur compréhension des demandes liées à l'islam. De fait, les institutions répondant à ces demandes se multiplient, principalement à destination des touristes musulmans, qui sont considérés comme une source de revenus importante pour l'industrie touristique ${ }^{25}$. La

25. Par ex., une organisation coopérative de collectivités locales a rendu un rapport expliquant comment attirer des touristes musulmans. V. THE JAPAN COUNCIL OF LOCAL AUTHORITIES FOR INTERNATIONAL RELATIONS SingAPORE, «Isuramuken karano kankôkyaku yuuchi - Tônan 
nécessité de prendre des dispositions en faveur des musulmans s'impose dans un contexte où le nombre de passagers venant de l'Asie du Sud-Est augmente de manière notable. Un chercheur estime ainsi que 24 millions de personnes ont visité le Japon en 2017, dont environ 950000 musulmans, soit $4 \%$ du total ${ }^{26}$. Si cette proportion de visiteurs musulmans ne semble pas significative, le gouvernement prévoit que le nombre de touristes musulmans s'accroîtra et que le marché adressé aux musulmans constitue un domaine d'expansion pour le développement du tourisme.

Dans ce contexte, en 2015, l'Office national du tourisme japonais a rédigé un guide d'accueil des musulmans afin de fournir aux restaurants et aux hôtels des informations pratiques sur l'islam et les mesures possibles pour satisfaire aux demandes religieuses. À cela s'ajoutent plusieurs collectivités locales qui ont commencé à soutenir financièrement des mesures en direction des touristes musulmans dans le but d'améliorer leurs conditions d'accueil. Les mesures varient selon les collectivités locales, mais la façon dont elles sont mises en balance avec le principe de séparation n'en reste pas moins discutable. Alors qu'il n'existe actuellement aucune jurisprudence relative au sujet, il est possible d'esquisser des pistes à travers quelques exemples. Ces exemples concrets permettent de mettre en lumière un processus des décisions administratives et de montrer de quelle manière le principe de séparation pourrait assurer la liberté de religion des minorités en conformité avec la Constitution.

\subsection{LE FINANCEMENT PUBLIC POUR LE TOURISME EN FAVEUR DES MUSULMANS}

Le premier exemple concerne un financement public visant à encourager l'obtention du certificat halal. En 2015, Taitô, un des arrondissements de Tokyo, a versé des subventions pour les restaurants de ce district qui s'efforçaient d'obtenir le certificat halal délivré par des institutions désignées. Cependant, l'obtention de ce certificat exige environ 800 euros au minimum pour passer l'examen, avec des frais supplémentaires pour les éventuels travaux nécessaires. Face à ce problème, Taitô a alloué une subvention pour l'obtention du certificat. À la suite de ce projet de financement, le nombre de restaurants ayant obtenu le certificat est passé d'un à vingt-trois en 2019.

asia no Musurimu kankôkyaku wo Nihon he» [Attirer les touristes musulmans - les touristes musulmans d'Asie du Sud-Est au Japon], Clair Report, no 393, 10 mars 2014, disponible en japonais: www.clair.or.jp/j/forum/pub/docs/393.pdf [consulté le 7 déc. 2020]. 26. R. Namikawa, Hararu Seihin [Produits halal], Tokyo, Soutensha-Shuppan, 2019, p. 255. 
Ce projet vise, bien entendu, à encourager les mesures favorables aux touristes musulmans. La question est de savoir si ce financement public est conforme au principe de séparation. Lorsque le bureau du tourisme de la municipalité de Taitô avait déposé ce projet, il a souligné que les problèmes principaux étaient un manque de produits halal et d'équipements pour la prière. Ainsi, il cherchait à prendre les mesures nécessaires sans déroger au principe de séparation. Le projet a été ensuite examiné par la commission spéciale de la culture et du tourisme de Taitô. La commission a conclu que la mise en place d'une allocation destinée à l'obtention du certificat halal ne serait pas inconstitutionnelle, mais qu'une subvention ayant pour but d'encourager la prière le serait, selon le principe de séparation ${ }^{27}$. Le bureau du tourisme explique que l'objet du projet est fondamentalement le développement du tourisme et que l'effet de son assistance ne dépasse pas la limite raisonnable. Le projet de financement a été finalement approuvé par le conseil municipal sans contestation.

Prenons un autre exemple, qui concerne également un projet de financement public au bénéfice des touristes musulmans: dans la préfecture de Gifu, située au centre du Japon, le département du commerce et de l'industrie a élargi, depuis 2018, le champ de son financement public dans le but d'améliorer le système d'accueil des touristes, en faveur par exemple de l'installation $\mathrm{du}$ wifi et du paiement par carte bancaire ${ }^{28}$. On trouve également dans ce projet une subvention visant à améliorer la capacité d'accueil des touristes musulmans, d'environ 1600 euros, au bénéfice de ceux qui se préparent à les accueillir, par exemple, des propriétaires de restaurants ou d'hôtels. Le financement par la préfecture concerne toute installation nécessaire pour s'adapter à l'accueil des visiteurs musulmans telle que la rénovation des équipements de cuisine, la création d'une salle de prière ou d'un espace d'ablution. La subvention peut aussi être destinée aux équipements nécessaires à la prière, comme un tapis de prière et des boussoles Qibla. Dans cet exemple, il semble que ce programme apporte une assistance à une religion particulière. Le département du commerce et de l'industrie explique

27. Actes du Conseil municipal de Taitô: Commission spéciale de la culture et du tourisme, organisée le 15 juin 2015. Dans cette commission, le chef du bureau du tourisme a déclaré: «La prière. Finalement le repas et la prière, c'est le plus grand problème pour accueillir les touristes musulmans. Nous, en tant que municipalité, nous lançons des mesures répondant aux demandes d'accès au halal pour la partie repas, mais pour la partie prière, le principe de séparation entre l'État et les religions s'avère une barrière » [traduit du japonais par l'auteur].

28. Gifu Prefecture Public Relations Division, «Announcements from Gifu Prefecture», May 2018. 
toutefois que ce financement fait partie du programme visant à la promotion du tourisme d'une manière générale et qu'il n'a pas pour but de viser une seule religion, mais tous les touristes. De plus, le département suppose que les receveurs des fonds sont des hôtels ou des restaurants non religieux et ce financement n'a pas en conséquence de but religieux ni n'apporte une assistance religieuse. Il insiste sur le fait que le premier objectif de la subvention est d'améliorer la commodité des visiteurs ${ }^{29}$. En effet, aucun débat relatif au principe de séparation n'a eu lieu à l'occasion de l'adoption du projet. L'explication du département semble toutefois chercher à atténuer l'effet d'une assistance religieuse. Il reste douteux que cette justification puisse éliminer un caractère d'assistance religieuse des financements. Bien que son premier objectif soit la promotion du tourisme d'une manière générale, la destination des financements, la création d'une salle de prière par exemple, peut apporter une assistance à des religions par son effet.

Un troisième cas montre une interprétation plus stricte du principe de séparation, tout en rendant possible l'aide financière au bénéfice des touristes musulmans. Depuis 2018, le JNTO a inauguré un programme de financement visant à améliorer les conditions d'accueil des touristes, pour que les demandes religieuses et culturelles des visiteurs étrangers soient prises en compte. Il correspond donc au type de projets mentionnés plus haut. Ce qui le distingue, ce sont les limites qu'il met au projet de financement pour répondre aux demandes religieuses, au regard du principe de séparation. Le projet explicite que l'objet du financement portera sur l'organisation de séminaires et de tournées d'inspection de lieux effectuant les mesures au bénéfice des touristes étrangers, qui permettent d'approfondir les connaissances pratiques sur les demandes des touristes, sous condition que les projets n'aient pas pour objectif de promouvoir une religion particulière. Le JNTO considère que le financement public doit être proportionné au principe de séparation et se réfère implicitement au critère de l'objectif et de l'effet. En conséquence, alors que des travaux d'adaptation aux demandes culturelles ou touristiques, par exemple la rénovation des toilettes et l'installation du wifi peuvent jouir d'aides financières, l'investissement répondant aux demandes religieuses se limite aux frais des séminaires et des tournées d'inspection.

De toute façon, malgré les différences de niveaux de subvention, nous soulignons un point commun: le financement public pour les mesures en faveur des touristes musulmans est mis en place sous certaines conditions.

29. Préfecture de Gifu. Service de la promotion de stratégie touristique (communication personnelle, 19 août 2020). 
Lorsqu'il s'agit de financement pour les mesures qui semblent avoir une signification religieuse, les agents publics relativisent cette signification en mettant l'accent sur l'objectif de promotion du tourisme. De plus, ils insistent sur le fait que les destinataires des fonds publics ne sont pas des associations religieuses.

Ces trois exemples ne font pas l'objet de contentieux pour le moment; leur légitimité juridique est néanmoins encore incertaine. Comme nous l'avons évoqué dans la première partie, la présence du maire et l'utilisation de fonds publics à une cérémonie religieuse ont pu être jugées constitutionnelles par la Cour suprême, soulignant l'importance touristique de la cérémonie. Cela démontre qu'un acte de l'État ou des collectivités locales ayant des liens avec une religion peut être justifié par son objectif autre que religieux, si son effet ne dépasse pas la limite raisonnable. Dans ce contexte, dans les trois exemples mentionnés, l'État et les collectivités locales précisent que leur but est fondamentalement touristique et qu'ils ne dépassent pas la limite raisonnable. Ces interprétations s'appuient sur une forte reconnaissance des religions et de leur utilité économique, sans pour autant s'engager sur leur signification religieuse.

Il reste toutefois douteux que ces interprétations puissent justifier un financement public directement destiné à toutes les activités de groupes religieux, qu'ils soient majoritaires ou minoritaires. L'offre de fonds public pour des groupes religieux particuliers doit être justifiée par un objectif autre que religieux. Dans ce cas-là, les religions traditionnelles pourraient être plus avantagées que les religions des nouveaux arrivants, d'autant plus qu'elles se voient légitimées par leur patrimoine culturel. Par exemple, les frais de travaux de rénovation d'une ancienne maison traditionnelle japonaise (kominka), dont le propriétaire est une association religieuse (le sanctuaire Mitsumine Jinja), ont été payés par la municipalité d'Otaki, sans dérogation au principe de séparation ${ }^{30}$.

30. Cette offre de fonds publics est légitimée par son objectif de financement. Le tribunal de première instance de Saitama l'a déclarée constitutionnelle, en précisant que cette maison traditionnelle possédait des valeurs culturelles et constituait une ressource touristique, sans que la maison en elle-même n'ait de signification religieuse. Ainsi, ce financement n'a pas été considéré comme s'opposant au principe de séparation dans la mesure où son objectif est de rénover un lieu touristique et que son rapport avec le shintoïsme n'a pas dépassé la limite raisonnable. V. H. TAJIKA, «Hanrei Ni Okeru Seikyôbunri Gensoku» [Le principe de séparation dans la jurisprudence], Shuumu-Jihou, no 120, oct. 2015, p. 8. 


\section{CONCLUSION}

Nous avons montré que le modèle japonais de «laicité» appartient à une laïcité séparatiste si nous nous référons aux seuls textes constitutionnels. Cependant, l'application de facto du principe de séparation est plus souple grâce au critère de l'objectif et de l'effet.

Or, comment pourrait-on comprendre la différence subtile d'interprétation entre les collectivités locales et le JNTO? Alors qu'au niveau national, l'interprétation du principe de séparation est assez stricte, au niveau local celui-ci est interprété de manière plus souple. Cela explique l'incertitude du critère de l'objectif et de l'effet dans le modèle japonais de «laïcité» et il doit être utilisé au cas par cas dans les décisions de la Cour.

Quoi qu'il en soit, les mesures d'aménagement en faveur des touristes musulmans sont légitimées par les gouvernements à travers le critère de l'objectif et de l'effet dans la mesure où ils mettent l'accent sur leur objectif autre que religieux: le développement du tourisme ${ }^{31}$.

Il faut néanmoins souligner que ce modèle n'échappe pas à une difficulté immanente, qui s'inscrit dans le critère de l'objectif et de l'effet: celle de ne prendre en compte que les religions économiquement «utiles». Si l'aide financière au bénéfice de certaines religions est légitimée par le tourisme, c'est-à-dire considérée comme essentielle pour le développement socio-économique, l'égalité et la liberté d'autres religions pourraient être négligées. L'État finirait par financer et garantir la liberté aux religions ayant une utilité économique, mais pourrait fermer sa porte aux religions jugées non utiles. Par exemple, l'amélioration de la situation des musulmans qui vivent au Japon est encore limitée. La construction et la conservation des mosquées reposent sur des dons des fidèles et des ressources étrangères, alors que les monuments des religions traditionnelles peuvent jouir d'aides financières en tant que monuments historiques. Pour des raisons économiques encore, les musulmans habitant au Japon n'ont pas la possibilité d'offrir à leur enfant une éducation islamique satisfaisante ${ }^{32}$. Les mesures au profit des minorités

31. Il faut toutefois rappeler que cela ne supprime pas tous les obstacles pour réaliser un tel dispositif. Dans la ville d'Ise en 2018, un projet de mise à disposition d'une salle à usages multiples, dont les usagers ne sont pas que musulmans, a été annulé, en raison de l'opposition d'habitants. Finalement cette salle a été installée sans l'équipement pour la prière.

32. H. Tanada, H. OkaI, « Nihon no Islâmu - Muslim community no Genjô to Kadai » [L'islam du Japon, la situation actuelle et les défis de la communauté musulmane], Shuumu-Jihou, $\mathrm{n}^{\circ} 119$, mars 2015, p. 1-22. 
religieuses ne sont pas encore suffisamment prises par le gouvernement japonais, alors que certains aménagements sont réalisés à l'égard des touristes.

Dans cette logique, le modèle japonais de «laïcité » ne garantit la liberté de religion que de manière partielle, négligeant la neutralité confessionnelle ${ }^{33}$. Il faut mettre en balance ces mesures au bénéfice des minorités religieuses avec le principe de séparation, tout en assurant le droit à la liberté de religion de tous, qui est un principe central de la Constitution.

33. D. Koussens, op. cit., p. 57. 